\title{
Schmerzpatienten in Bewegung bringen
}

\author{
Martin Lotze und G. Lorimer Moseley
}

\begin{abstract}
Das komplexe regionale Schmerzsyndrom (CRPS) ist durch unerträgliche und meist wechselnde, brennende oder ziehende Schmerzen charakterisiert. Etwa 65000 Patienten sind in Deutschland von dieser Krankheit betroffen. Eine wirksame Strategie ist das stufenweise Imaginationstraining (GMI).
\end{abstract}

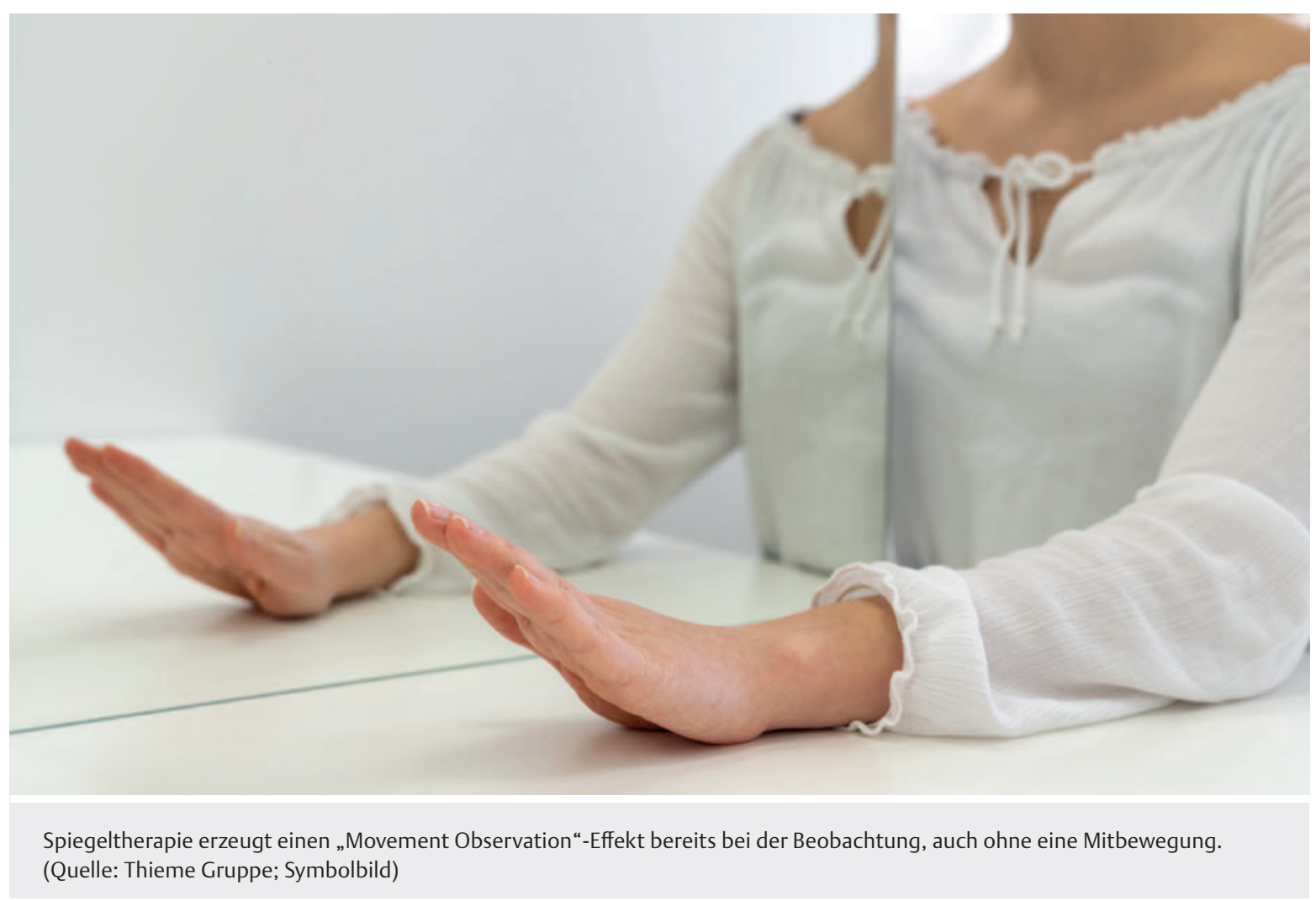

Man kann chronische Schmerzen insgesamt als einen pathologisch außer Kontrolle geratenen Prozess des zentralen Nervensystems ansehen mit einer - zumindest in frühen Stadien - Beteiligung des Immunsystems. Bei neuropathischen Schmerzen wie dem CRPS kann man über der Hemisphäre kontralateral der betroffenen Extremität eine erhöhte Erregbarkeit des primären sensomotorischen Kortex sowie veränderte Repräsentationsfelder in gleichen Gehirnarealen beobachten. Diese neurophysiologischen Veränderungen des Gehirns sind oftmals mit klinischen Symptomen assoziiert (Übersicht in [9]). Hierzu zählen außer der geschilderten Schmerzsymptomatik eine Allodynie (auch sehr leichte Berührungen werden als schmerzhaft gewertet), Verminderung der räumlich-somatosensorischen Auflösung, Dystonien, Tremor, aber auch neuropsychologische Defizite [18].

Es gibt eine Reihe von Strategien, um diese maladaptiven Prozesse wieder rückgängig zu machen. Die meisten wirksamen Verfahren versuchen, durch den zunehmenden Einsatz der betroffenen Extremität (der Einfachheit halber sprechen wir jetzt hier von der betroffenen Hand) physiologische Repräsentationsmuster zu induzieren. Der Schmerz jedoch bringt den Patienten immer wieder dazu, 


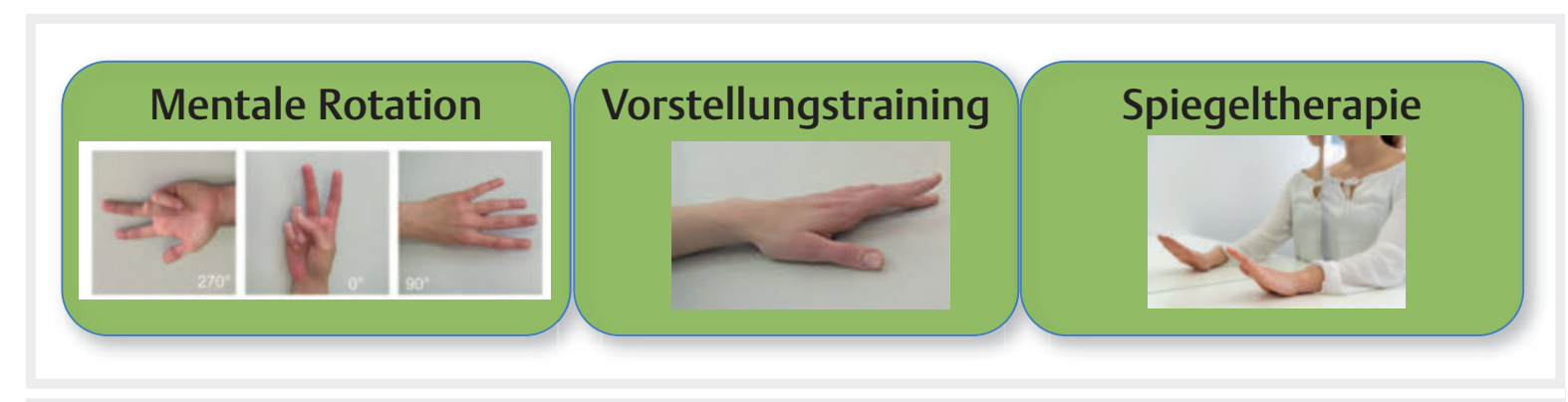

Abb. 1 GMI in den drei Stufen: mentale Rotation; Vorstellungstraining und Spiegeltherapie. Jede Stufe wird für zwei Wochen für für 5-10 Minuten in jeder Wachstunde der Wachphasen trainiert [11]. (Quelle: M. Lotze, li./Mitte, und Thieme Gruppe; re.)

jegliche Bewegung zu vermeiden. Aus diesem Grund wurden Strategien entwickelt, um den Patienten in die Bewegung schrittweise hineinzuführen. Eine wirksame Vorgehensweise ist das stufenweise Imaginationstraining (graded motor imagery, GMI) [11].

\section{Geschichte der GMI}

Vor 25 Jahren publizierte R. Ramachandran einen viel beachteten Artikel über einen Patienten mit Phantomschmerzen nach traumatischer Amputation der oberen Extremität, bei dem durch ein Spiegeltraining erfolgreich die Phantomschmerzen gelindert werden konnten [17].

Bei der Beobachtung der intakten Hand bei Durchführung einer Bewegung, gespiegelt auf die Seite der fehlenden Extremität, erhält der Patient die dominierende visuelle Rückmeldung, dass die fehlende Extremität eine intakte Bewegung durchführt. Gelingt diese Illusion, so kann dadurch ein zumindest kurzfristiger lindernder Effekt auf die Phantomschmerzen erreicht werden. Interessant ist dies vor allem deshalb, weil es einiges über die Repräsentation unseres Körpers aussagt: Das Gehirn führt alle Sinnesmodalitäten zu einer einheitlichen Körperrepräsentation (body matrix; [14]) zusammen. Da hierbei die visuelle Information dominant ist, wird die Ansicht der sich bewegenden gesunden Hand auf die betroffene Seite gespiegelt. Damit entsteht die Illusion, dass sich die betroffene Hand wie eine gesunde Hand bewegen kann. Diese „Spiegelillusion" funktioniert, obwohl wir von unseren propriozeptiven Informationen eine statische Haltung unserer Hand rückvermittelt bekommen.

Schon sehr früh war festgestellt worden, dass sich die Körperrepräsentation im Parietallappen befinden muss. So hat Gerstmann bereits 1930 als Ursache für einen Neglekt eine Läsion im Parietallappen kontralateral zur vernachlässigten Seite beschrieben. Die dort lokalisierte Repräsentation des Körpers ermöglicht es, dass Objekte ohne zusätzliche Überprüfung (etwa durch den Blick auf den Arm) weitgehend implizit korrekt ergriffen werden können.

Man kann die Körperrepräsentation jedoch auch durch propriozeptive Reize beeinflussen: Vibrationsreize über dem Bizepsmuskel vergrößern die gefühlte Armlänge, und beim gleichzeitigen Ergreifen der Nase scheint der gesamte Kopf gefühlt mitzuwachsen [6]. Zudem ist die Repräsentation des eigenen Körpers mit der Wahrnehmung von Körperteilen anderer Personen verbunden: Besteht etwa ein Neglekt bei rechtsseitiger Läsion zur linken Körperseite, so werden in der Handerkennung rechte Hände schneller erkannt als linke Hände [2]. Dieses charakteristische Muster wurde ebenfalls bei Patienten beobachtet, die unter chronischem CRPS leiden [19].

Aus einem verhaltenstherapeutischen Ansatz heraus sieht man die Angst vor Bewegungsschmerz und dem Vermeiden von Bewegung als ein „fear avoidance model“ (Übersicht in [8]), das wie andere Ängste in einer Expositionstherapie behandelt werden kann [20]. Tatsächlich ist diese Expositionstherapie wirksam. Eine stufenweise Erhöhung der Bewegung kann dabei die Patienten schrittweise in die Bewegung einführen. Mentales Training ist hierfür eine ideale Möglichkeit, weil sich bei der Bewegungsvorstellung Repräsentationen im Gehirn zum Teil mit denen bei tatsächlicher Bewegung überlappen [7].

\section{Darstellung der Durchführung der GMI}

- Abb. 1 zeigt einen Überblick über das stufenweise Imaginationstraining GMI.

Die GMI [11] wurde auf folgender Basis entwickelt:

- Die bestehenden Daten von Neglekt-Patienten wiesen auf eine schlechtere Leistung in der mentalen Rotation von Händen auf der betroffenen Körperseite hin.

- Klinische Beobachtungen zeigten, dass eine Spiegeltherapie allein sich nicht als wirkungsvoll in der Behandlung der CRPS-Schmerzen erwies.

- Eine Stimulusreduktion (Bewegung) führt nicht mehr zu einer Schmerzreduktion bei chronischen Schmerzpatienten, sodass eine stufenweise Konfrontation mit Bewegung - ähnlich wie in der Verhaltenstherapie [20] - hier insgesamt zu einer Verbesserung von Schmerz und dem Einsatz der Extremität führen kann. 
Implizite Bewegungsvorstellung Bei der mentalen Rotationsaufgabe von Bildern von Händen soll der Proband lediglich angeben, ob es sich um eine rechte oder linke Hand handelt (Handlateralisationsaufgabe). Dabei wird der Proband - ohne es zu wissen, also implizit - seine eigene Hand so versuchen zu drehen, bis sie auf die dargestellte Hand passt. Da bereits eine vorgestellte Bewegung der betroffenen Hand bei CRPS-Patienten Schmerzen auslöst, nutzt man den Umstand dieser implizit vorgestellten Handbewegung, um Bewegungssteuerungsfelder im Gehirn zu aktivieren.

Mit der gesunden Hand wird nach jeder präsentierten Hand die Einschätzung der Seite (links oder rechts) möglichst schnell angegeben. Dadurch kann nach jedem Block die Anzahl der richtigen Lösungen sowie die durchschnittliche Zeit zur Entscheidung dargestellt werden. Die Handlateralisationsaufgabe bildet den ersten zweiwöchigen Schritt der GMI-Therapie.

Explizite Bewegungsvorstellung Der zweite Schritt ist die explizite Bewegungsvorstellung. Hier soll gedanklich die Handstellung eingenommen werden, die auf Bildern von Händen dargestellt wird. Dabei wird immer die betroffene Seite trainiert. Hier ist kein direktes Feedback möglich, der Patient erfährt jedoch oftmals zunächst eine Intensivierung der Schmerzen. Um diese Belastung niedrig, aber die Aufmerksamkeit maximal zu halten, sind die Übungssessions immer nur von kurzer Dauer (abhängig von der Schnelligkeit des Patienten im Mittel bei 5-10 Minuten), werden aber - ebenso wie der Schritt mit der Handlateralisationsaufgabe - möglichst in jeder Stunde des Tages wiederholt.

Spiegeltherapie Der dritte Schritt ist die Spiegeltherapie, die als alleinige Therapie nur eine geringe Evidenz zeigt, den Schmerz oder die Armfunktion bei CRPS zu verbessern [1]. Der Patient erhält bei Bewegung der gesunden Hand das visuelle Feedback (gespiegelt) einer physiologischen Bewegung seiner kranken Körperseite. Dadurch besteht ein „Movement Observation“-Effekt bereits bei der Beobachtung auch ohne eine Mitbewegung, und zudem wird ein Feedback von Schädigungen der kranken Seite vermieden. Die Aufgabe für den Patient ist es, im Laufe der Sitzungen immer mehr auch die kranke Seite mit zu beteiligen - bilaterale Bewegungen. Nach diesen letzten zwei Wochen Therapieblock ist die GMI abgeschlossen, und der Patient bekommt die Instruktion, jetzt die betroffene Hand bei Tagesaktivitäten immer mit einzusetzen.

Allgemeine Hinweise Wesentlich für verhaltensrelevante Therapien sind

- das häufige Üben (Repetition),

- das Üben an der individuellen Leistungsgrenze (Shaping) und

- die Leistungskontrolle nach jedem Übungsschritt (Feedback).
Eine kommerziell erhältliche App (noi-group; www. noigroup.com/shop/?cf=32) für Tablets, Laptops oder Smartphones ermöglicht dem Patienten das Training der Stufe 1 und 2 zu Hause. Die Spiegeltherapie wird mit einem klappbaren Folienspiegel ebenfalls zu Hause durchgeführt.

\section{GMI und Trainingsmotivation}

Leider ist dieser Prozess aufwendig: Der Patient wird instruiert, jede der drei Stufen der GMI an jedem Tag stündlich zu den Wachzeiten für die Dauer der Einzelsession durchzuführen. Hierbei wird erfahrungsgemäß erst ab etwa sieben Sitzungen pro Tag eine relevante Verbesserung der Schmerzsymptomatik auch im chronischen Stadium der CRPS erzielt. Es ist also entscheidend, wie gut der Therapeut den Patienten motivieren kann, dass sich der Aufwand für ihn lohnt, und wie gut die Anbindung an den Therapeuten ist, wenn es zu Trainingsproblemen kommt. Über die Onlinestatistik kann der Therapeut die Trainingszeiten und Trainingserfolge jederzeit abrufen und ggf. rasch intervenieren. Dieses hohe Niveau der extrinsischen Motivation und Kontrolle könnte sehr wohl für einen großen Anteil der Effektivität des Trainings verantwortlich sein.

Ist die Folge der Therapiestufen entscheidend? Natürlich ist das Modell der stufenweisen Einführung in die Bewegung über implizite und explizite Vorstellung sowie der beobachteten Handbewegungen der gesunden Hand auf der Seite der betroffenen Hand für das schrittweise Hineinführen in die Bewegung der betroffenen Hand vom Konzept her entscheidend. Und tatsächlich konnte gezeigt werden, dass diese Folge der Therapieschritte besonders wirkungsvoll ist [12].

\section{Evidenzlage und Therapie- empfehlungen bei CRPS}

Insgesamt ist die Evidenzlage für die GMI derzeit günstig (Cochrane Review: [15]), obwohl noch größere Multicenter-Untersuchungen ausstehen und die zur Verfügung stehenden Daten vor allem durch eine Gruppe (um den Autor G. L. Moseley) dominiert werden. Die GMI wird derzeit in den Leitlinien von einigen Ländern wie England, USA und den Niederlanden empfohlen (für die Niederlande siehe [16]).

Biomarker Die Autoren und ihre Teams können zudem zeigen, dass sich auch die neurophysiologischen Pathologien, wie die verminderte kontralaterale kortikale Inhibition im primären Motorkortex, durch die Therapie günstig beeinflussen lassen. Dennoch wird in den deutschsprachigen Ländern die GMI immer noch zu wenig angeboten, und Patienten nehmen oft große Entfernungen in Kauf, um in Studien an der Therapie teilnehmen zu können. Da die maladaptiven Veränderungen, wie verminderte kortikale Inhibition oder das Schrumpfen der Handrepräsen- 
tation, zumindest in einigen Studien deutlich mit den klinischen Pathologien assoziiert sind, bieten sich diese Parameter auch als Biomarker an, um frühzeitig besonders gute und schlechte Therapieresponder zu ermitteln. Da es hier - trotz der im Durchschnitt signifikanten Effekte insbesondere auf den Bewegungsschmerz - noch deutliche Unterschiede in den Verläufen gibt, sind Biomarker notwendig.

Modulation der Therapie Zudem könnten die longitudinalen Untersuchungen mit Darstellungen der Biomarker unser Wissen zu der Modulation der Therapie etwa durch sensorisches Diskriminationstraining [13] oder medikamentöse Strategien [4] hinsichtlich der zugrunde liegenden Mechanismen erfassen und so den Hauptnachteil der Therapie, den großen Einsatz von Zeit und Motivation des Patienten, günstig modulieren.

\section{Autorinnen/Autoren}

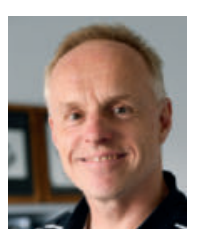

\section{Prof. Dr. Martin Lotze}

Professor für Funktionelle Bildgebung und Neurologie an der Universität Greifswald. Er forscht unter anderem über neuronale Effekte bei Training, neuronale Plastizität und chronische Schmerzen.

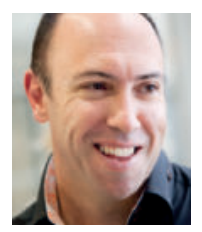

\section{Prof. Dr. G. Lorimer Moseley}

Lorimer Moseley ist Professor für Clinical Neuroscience und Chair in Physiotherapy an der University of South Australia. Sein Interesse gilt der Schmerzforschung.

\section{Korrespondenzadresse}

Prof. Dr. Martin Lotze

Ernst-Moritz-Arndt-Universität Greifswald

Walter-Rathenau-Str. 46

17475 Greifswald

E-Mail: martin.lotze@uni-greifswald.de

\section{Literatur}

[1] Bowering KJ, O'Connell NE, Tabor A et al. The effects of graded motor imagery and its components on chronic pain: A systematic review and meta-analysis. The Journal of Pain: Official Journal of the American Pain Society 2013; 14: 3-13

[2] Coslett HB. Evidence for a disturbance of the body schema in neglect. Brain Cogn 1998; 37: 527-544

[3] Gerstmann J. Zur Symptomatologie der Hirnläsionen im Übergangsgebiet der unteren Parietal- und mittleren Occipitalwindung. Nervenarzt 1930; 3: 691-695
[4] Gustin S, Schwarz A, Birbaumer N et al. NMDA-antagonist and morphine decrease CRPS-pain and cerebral pain-representation. Pain 2010; 151: 69-76

[5] Harden RN, Oaklander AL, Burton AW et al. Complex regional pain syndrome: Practical diagnostic and treatment guidelines. 4th ed. Pain Medicine (Malden, Mass.) 2013; 14: 180-229

[6] Lackner JR. Some proprioceptive influences on the perceptual representation of body shape and orientation. Brain 1988; 111: 281-297

[7] Ladda AM, Lotze M. Mentales Training und Bewegungsbeobachtung. neuroreha 2019; 11: 118-122

[8] Leeuw M, Goossens ME, Linton SJ et al. The fear-avoidance model of musculoskeletal pain: Current state of scientific evidence. J Behav Med 2007; 30: 77-94

[9] Lotze M. Maladaptive Plastizität bei chronisch neuropathischen Schmerzen. Der Schmerz 2015; 30: 127-133

[10] McCabe CS, Haigh RC, Ring EF et al. A controlled pilot study of the utility of mirror visual feedback in the treatment of complex regional pain syndrome (type 1). Rheumatology (Oxford, England) 2003; 42: 97-101

[11] Moseley GL. Graded motor imagery is effective for longstanding complex regional pain syndrome: A randomised controlled trial. Pain 2004; 108: 192e198

[12] Moseley GL. Is successful rehabilitation of complex regional pain syndrome due to sustained attention to the affected limb? A randomised clinical trial. Pain 2005; 114: 54-61

[13] Moseley GL, Zalucki NM, Wiech K. Tactile discrimination, but not tactile stimulation alone, reduces chronic limb pain. Pain 2008; 137: 600-608

[14] Moseley GL, Gallace A, Spence C. Bodily illusions in health and disease: Physiological and clinical perspectives and the concept of a cortical "body matrix". Neuroscience and biobehavioral reviews 2012; 36: 34-46

[15] O'Connell NE, Wand BM, McAuley J et al. Interventions for treating pain and disability in adults with complex regional pain syndrome. Cochrane Database Syst Rev 2013; 4: CD009416

[16] Perez RS, Zollinger PE, Dijkstra PU et al. Evidence based guidelines for CRPS type 1. BMC Neurology 2010; 10: 20-60

[17] Ramachandran VS, Rogers-Ramachandran D, Cobb S. Touching the phantom limb. Nature 1995; 377: 489-490

[18] Reid EJ, Braithwaite FA, Wallwork SB et al. Spatially-defined motor deficits in people with unilateral complex regional pain syndrome. Cortex 2018; 104: 154-162

[19] Schwoebel J, Friedman R, Duda N et al. Pain and the body schema: Evidence for peripheral effects on mental representations of movement. Brain 2001; 124: 2098-2104

[20] Vlaeyen JW, Linton SJ. Fear-avoidance and its consequences in chronic musculoskeletal pain: A state of the art. Pain 2000; 85: 317-332

Bibliografie

DOI https://doi.org/10.1055/a-1156-3958 neuroreha 2020; 12: 82-85

(c) Georg Thieme Verlag KG Stuttgart · New York ISSN 1611-6496 